\title{
RESTORING THE SKEW QUADRUPOLE MOMENT IN TEVATRON DIPOLES*
}

\author{
D.J. Harding\#, P.C. Bauer, J.N. Blowers, J. DiMarco, H.D. Glass, R.W. Hanft, J.A. Carson, \\ W.F. Robotham, M.A. Tartaglia, J.C. Tompkins, G. Velev
}

Fermilab, IL 60510, U.S.

\section{Abstract}

In early 2003 it was realized that mechanical changes in the Tevatron dipoles had led to a deterioration of the magnetic field quality that was hindering operation of the accelerator. After extensive study, a remediation program was started in late 2003 that will continue through 2005. The mechanical and magnetic effects are discussed. The readjustment process and experience are reported, along with other observations on aging magnets.

\section{INTRODUCTION}

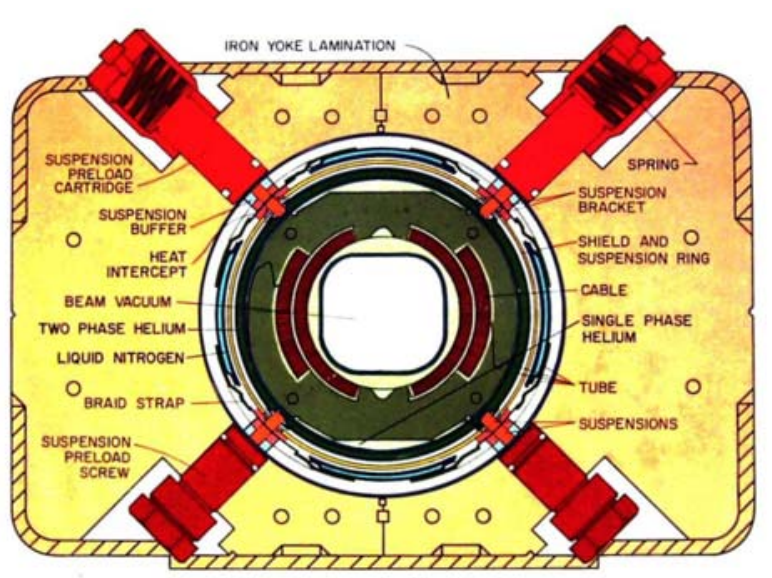

Figure 1 Tevatron dipole cross section

In January 2003 two lines of inquiry converged, leading to the recognition that the severe betatron coupling that was hindering operation of the Tevatron could be explained by a systematic shift on the skew quadrupole field in the dipole magnets of the same size expected from observed mechanical movement of the coils inside the magnet yokes. [1] This paper reports on subsequent magnet studies that were conducted in parallel with additional beam studies and accelerator modeling [2] exploring the feasibility of the eventual remediation effort. [3]

\section{MECHANICAL ISSUES}

The Tevatron dipole cross section is shown in Figure 1. A warm iron yoke surrounds the cryostat tube and the collared coil within it. The collared coil is supported in the cryostat at nine stations down the 6-meter length of the magnet. At all but the center station the support is two rectangular blocks of G11, called "suspensions", approximately $8.5 \mathrm{~mm}$ x $6.4 \mathrm{~mm}$ x $17 \mathrm{~mm}$, aligned with

* Work supported by the U.S. Department of Energy under Contract No. DE-AC02-76CH03000

"harding@fnal.gov each other, one between the outer cryostat skin and the heat intercept at liquid nitrogen temperature and the other between the heat shield and the phase single helium tube. These suspensions are arranged in grooves that allow the whole cryostat to move relative to the yoke as it is cooled. At the middle station more robust G10 tubes, known as "anchors" support the cryostat, anchoring the center against both longitudinal motion and rotation relative to the yoke (a bane of earlier designs of the magnet).

At each station four supports are located at the 45 degree points. A screw or suspension cartridge through the yoke at each of these points contacts the outside of the cryostat. This allows the cryostat to rest stably in a cradle formed by the lower two screws ("bolts"), while being held down against those supports by the upper two suspension preload cartridges, springloaded cartridges known as "smart bolts." The pre-loaded springs maintain a force as the collared coil and cryostat shrink during cool down.

The sizing of the G11 suspensions was a balance between mechanical strength and the heat load a larger conducting cross section would present. The stability of the blocks was the subject of a study at the time [4]. One participant remembers the 1980 guidance to "design the magnets to last for 20 years" [5].

Recognizing the need to monitor the position of the coil during the shimming operation and the potential for “creep", a long-term deformation of the blocks under constant pressure, provision was made to measure the extension of the cylinders in the smart bolts. A depth gauge can be used to measure the distance from the top of the cylinder to the top of the cartridge, a distance that became known as the "lift". For example, as the magnet was cooled, the collared coil shrank, the outer skin of the cryostat deformed slightly, the springs pushed the cylinders inward, and the lift increased.

As part of the feasibility study after the possible problem was identified, additional magnets were measured in the tunnel during a short down period in the spring of 2003. Many more magnets have since been measured during the major shutdowns of 2003 and 2004. The distribution of lift change, averaged over each magnet, is plotted in Figure 2. The average shift of about $0.14 \mathrm{~mm}(0.0055$ ”) is clearly seen, as is the breadth of the distribution. The difference between the average left and right lift changes is centered on zero, indicating no lateral movement. 


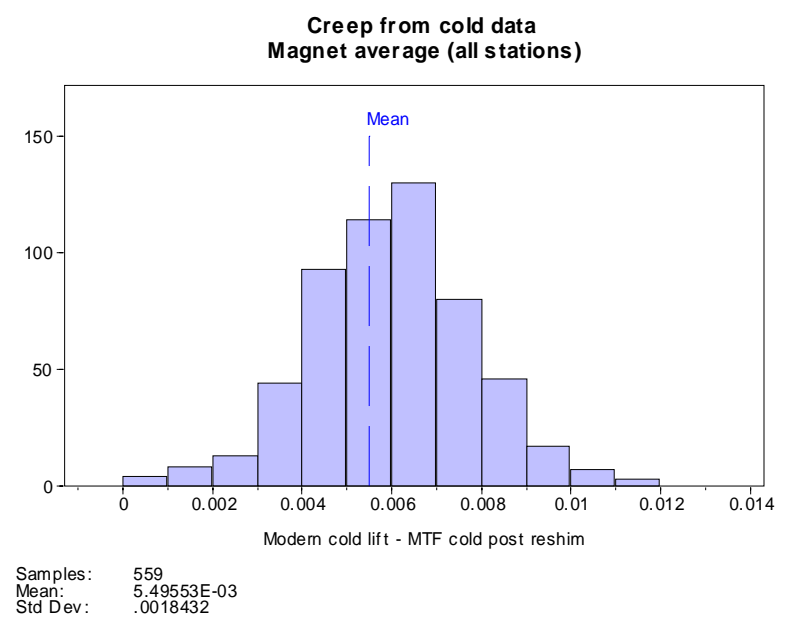

Figure 2 Distribution of lift changes from production to now due to creep. For each magnet the change is averaged over all accessible stations.

The spring force in the smart bolts is sufficiently greater than the weight of the cryostat that we take the compressive force on the upper and lower suspensions to be equal and interpret the change in lift to be creep equally distributed between the upper and lower sets. Note also that a equal creep all around results in a change in the coil position that is the vector sum of the creep in the two lower suspensions.

\section{MAGNET FIELD CORRECTION}

When there is any asymmetry in the coils of a conductor-dominated dipole magnet, an error field is produced, which we characterize by the coefficients of its harmonic decomposition. For convenience we normalize the error field to the central field, take the reference radius to be $25.4 \mathrm{~mm}$, and quote the coefficients in "units" of parts in $10^{4}$. This means that if we can bring the coefficients in to the range unity, the magnetic field will be acceptable over the useful aperture of the magnet.

The asymmetry may be in the geometry of the coils, in their placement in the yoke, or in the superconducting cable. Of particular concern here is the fact that a normal (skew) quadrupole term is produced by the horizontal (vertical) displacement of the coils from the center with a linear dependence. By adjusting the cryostat and collared coil position in the yoke, the quadrupole components of the field could be eliminated or cancelled during production.

The cryostat support system described above allowed these adjustments while the magnet was under test cold. During assembly brass shims were placed on the end of all four bolts at each station to roughly center the coil in the yoke. While the magnet was still on the test stand after an initial round of magnetic measurements, the bolts were removed one opposing pair at a time and shims were added or removed to reposition the coil, bringing both the normal and skew quadruple components of the field into tolerance.

\section{MAGNETIC FIELD ISSUES}

During production each magnet was measured in some detail. [6] After quench testing, the harmonic components of the magnet field integral were measured and the magnet was shimmed according to the guidance provided by a computer calculation. The magnet was then measured and multiple currents on both the up and down ramps. This data set has the advantage of including every magnet and of providing a measurement of the entire magnet field integral.

When the creep issue was recognized, a program of measurements was under way at the Fermilab Magnet Test Facility (MTF) studying the dynamic behavior of Tevatron dipoles. [7] The work was expanded to include some measurements related to the skew quadrupole questions. [8] Using modern techniques, these measurements can capture the field during a ramp at very densely spaced currents. However, only a small sample of spare magnets are available for measurement, not necessarily representative of the installed magnets. The modern probe is short $(0.83 \mathrm{~m})$ and the fixturing was inadequate to allow a reliable full length measurement to be assembled.

\section{Skew Quadrupole Distribution}

The specification called for both quadrupole components to be adjusted, if needed, to be within one unit of zero. Figure 3 shows the final distribution of the skew quadrupole component after a subsequent refinement of the data processing.

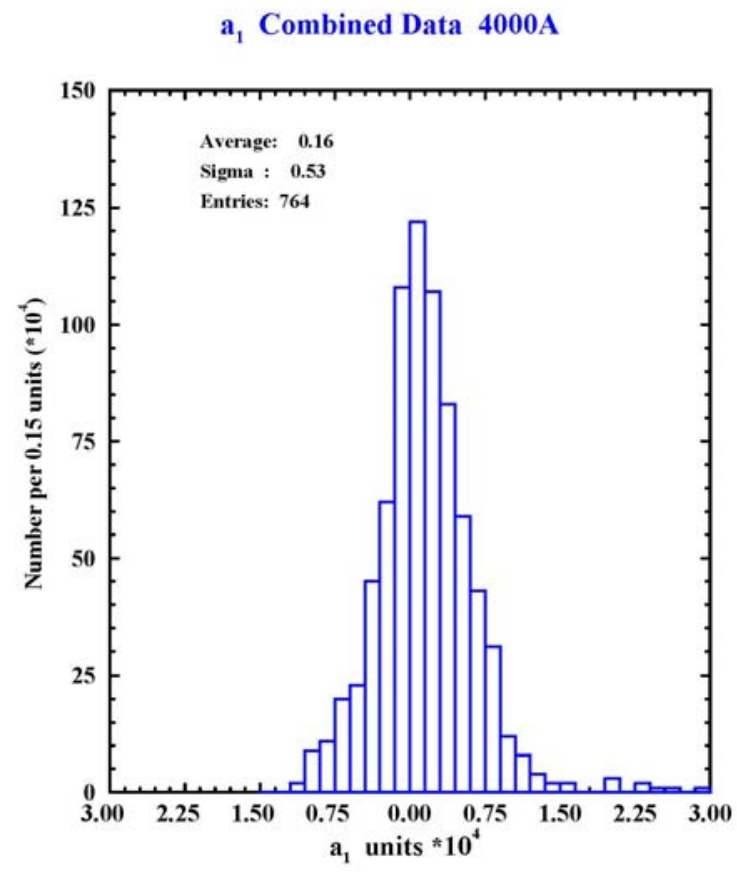

Figure 3 Final distribution of skew quadrupole in Tevatron dipoles after production shimming

Measurements of both the lifts and the fields of spare magnets confirmed that the lifts had increased consistent with the magnets in the tunnel and that the skew 
quadrupole had changed commensurately. The amount of its life that a magnet had spent cold did not seem to affect its creep.

\section{Incomplete Reshimming}

As part of the preparation for reshimming magnets in the tunnel, one dipole was reshimmed on the test stand at MTF while cold, with harmonic measurements before and after each step. Recognizing that the production experience in shimming close to a thousand magnets had validated the technique exhaustively, only a few people insisted on seeing another demonstration of the basic procedure. There were, however, a few more subtle questions.

Since it was anticipated that on many magnets one or more stations would be inaccessible due to residual Main Ring magnets stands and other interferences, especially at the ends, it was important to establish the behavior after non-uniform reshimming. The short probe of the modern system allowed measurement of the field as a function of position. Artifacts such as effects of the superconducting cable pitch confused these measurement, but in the end it was agreed that the as many as two stations could be left unshimmed without a significant impact on the integral.

\section{Skew Quadrupole Variation with Excitation}

Archival data showed that the skew quadrupole contribution of many magnets varied as a function of current, suggesting coil movement under excitation. (Figure 4) The effect was small and there were magnets that exhibited both signs of variation.

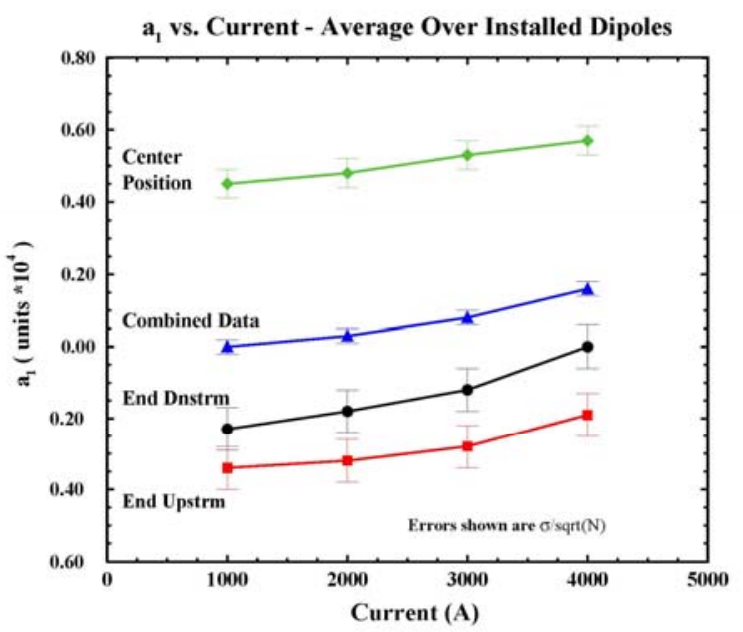

Figure 4 Current dependence of the average skew quadrupole for each third of the dipoles and the whole magnets ("combined")

One possible mechanism considered was that if the coil was too high, the decentering Lorentz force might overcome both gravity and the springs of the smart bolts. Before this phenomenon was recognized during production, two magnets were damaged. After a limit was put on the allowed coil position there was no further damage, but small movements were not ruled out. The modern MTF test did rule out coil motion. First, Gross motion was checked by measuring lifts during a full current ramp cycle. Then the coil was purposely positioned up, down, left, and right by far more than normal, exaggerating any decentering forces. Under those conditions no changes were observed in the quadrupole field as a function of the decentering force, as shown in Figure 5.

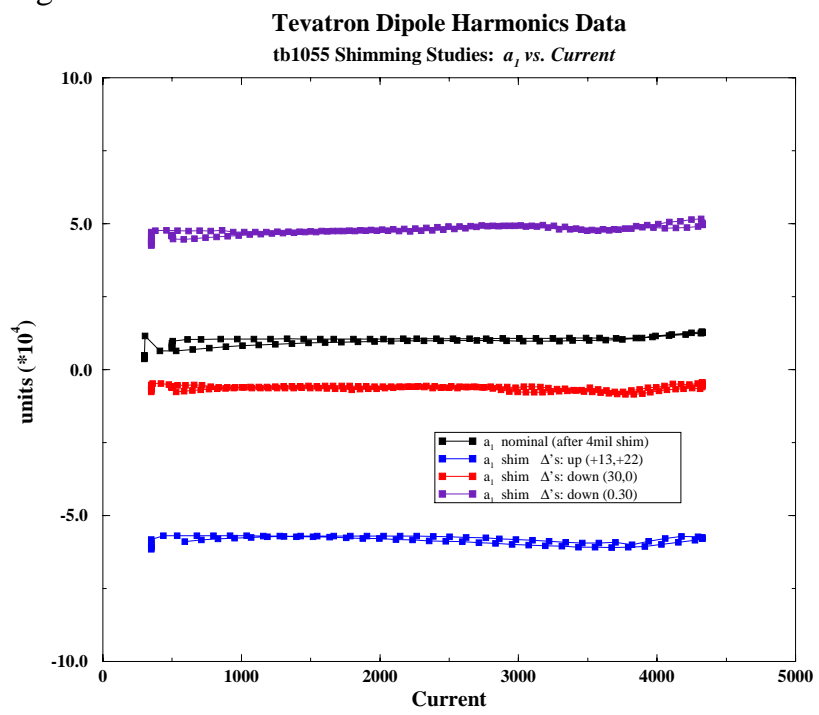

Figure 5 Skew quadrupole as a function of current for one magnet with the coil offset from center.

\section{CONCLUSIONS}

Extensive studies were conducted of the skew quadrupole in Tevatron dipoles, especially that due to creep in the cryostat suspension. The conclusion was that reshimming the magnets in the tunnel was a safe, reliable remedial action to a serious problem.

\section{REFERENCES}

[1] M.J. Syphers and D.J. Harding, "Deterioration of the Skew Quadrupole Moment in Tevatron Dipoles Over Time”, this conference.

[2] M.J. Syphers, et al., "Observations of Strong Transverse Coupling in the Tevatron”, this conference.

[3] J.N. Blowers, et al., "Reshimming of Tevatron Dipoles; A Process-Quality and Lessons-Learned Perspective”, this conference.

[4] Edward M. Caulfield, "Investigation of Creep Behavior of the Glass Reinforced Composite StandOffs,” Packer Engineering Associates Inc., 1981

[5] Gregory Kobliska, private communication

[6] R.W. Hanft, et al., "Magnetic Field Properties of Fermilab Energy Saver Dipoles”, Proceedings of PAC83.

[7] G. Velev, et al., "Measurements of Field Decay and Snapback Effect on Tevatron Dipole and Quadrupole Magnets", this conference.

[8] G. Ambrosio, et al, "Results Of An Investigation Of The Skew Quadrupole Issue In Tevatron Dipoles”, Fermilab note TD-03-045, 2003. 